standard, validated 10 observation right lobe ARFI technique. Within the study period, 82/108 chronic liver disease (CLD) patients (60 hospitalised and 48 outpatients) underwent both elastography and upper GI endoscopy. Standard US data collected included spleen size, portal vein Doppler flow velocity, direction and waveform, presence of collaterals, and platelet count.

Results Significant endoscopic PHT, defined as oesophageal/gastric varices and/or moderate to severe portal hypertensive gastropathy, was seen in $34 / 82$ patients (41\%). Median LS measured by shear velocity was significantly higher in the PHT group (2.9 vs $2.2 \mathrm{~m} / \mathrm{s}$, $\mathrm{p}<0.001)$. A 10 point "SPVD" (spleen-platelets/portal vein Doppler) scoring system, devised to include all US parameters and platelet count, showed significantly higher median scores in PHT (3 vs 1, $\mathrm{p}<0.001)$. Multiple logistic regression analysis demonstrated that both ARFI and SPVD score were independent predictors of PHT (OR, 95\% CI $2.73,1.22$ to $6.13, \mathrm{p}=0.02$; and $3.08,1.89$ to $5.02, \mathrm{p}<0.001$, respectively). AUROC analysis showed that a best fit combination of ARFI+SPVD score showed the highest overall predictive value at 0.91 , compared with ARFI or SPVD alone (0.72 and 0.87, respectively).

Conclusion In this retrospective "real world" study the addition of ARFI to standard US parameters using a combined scoring system achieved high $(>90 \%)$ predictive value for the non-invasive detection of endoscopic PHT. A further prospective study, with refinement of US Doppler technique, is now in progress to confirm these promising results. Single session ARFI+US may accurately guide PHT diagnosis and selection for endoscopic surveillance in CLD.

Competing interests None declared.

\section{PTU-026 PREVALENCE OF LIVER HISTOLOGICAL ABNORMALITIES IN TYPE 1 DIABETES AND THE LONG TERM CONSEQUENCES}

doi:10.1136/gutjnl-2012-302514c.26

${ }^{1} \mathrm{D}$ J Harman, ${ }^{*}{ }^{2} \mathrm{R}$ Harris, ${ }^{3} \mathrm{~A}$ Gazis, ${ }^{4} \mathrm{P}$ Kaye, ${ }^{1} \mathrm{G}$ Aithal. ${ }^{1}$ Hepatology, NIHR BRU, Nottingham, UK; ${ }^{2}$ General Medicine, Nottingham University Hospitals NHS Trust, Nottingham, UK; ${ }^{3}$ Endocrinology, Nottingham University Hospitals NHS Trust, Nottingham, UK; ${ }^{4}$ Histopathology, Nottingham University Hospitals NHS Trust, Nottingham, UK

Introduction Patients with type 1 diabetes have a higher prevalence of raised liver enzymes than the general population. Ultrasound diagnosis of non-alcoholic fatty liver disease has been reported to be common in type 1 diabetes despite associated insulin deficiency rather than insulin resistance. However, the histological spectrum of liver disease in type 1 diabetes and the natural history of chronic liver disease in this cohort is unknown. We describe the histological findings of patients with type 1 diabetes who had liver biopsy in a tertiary referral centre, and their long-term clinical outcome.

Methods The DIAMOND database, which contains longitudinal data for over $95 \%$ of type 1 diabetes patients from an overall catchment population of 750000 in Nottingham, was crossmatched with the clinical pathology database to identify those who had undergone liver biopsy. Case notes were reviewed to obtain followup data, and identify liver and non-liver related outcomes.

Results Out of 2800 patients with Type 1 Diabetes, 57 patients underwent a total of 82 liver biopsies. Common indications for biopsy were abnormal liver enzymes (28 patients), malignancy (8), Hepatitis C staging (7) and clinical evidence of cirrhosis (3). On index biopsy, $86 \%$ had significant histological abnormalities (Abstract PTU-026 figure 1) and 10 patients (17.5\%) had cirrhosis. During a total follow-up of 336.4 patient years (median 5.6 years), a further four patients developed cirrhosis, giving a cirrhosis prevalence of at least 500 per 100000 population - this compares with an estimated UK cirrhosis prevalence of 76.3 per 100000 population. ${ }^{1}$ Portal hypertensive sequelae occurred in 11 patients (78.6\%) with cirrhosis and hepatocellular carcinoma in three patients. 22 patients (38.6\%) died during follow-up. Crude death rate was 6539 per 100000 person years, compared with national Type 1 Diabetes data ${ }^{2}$ of 1878 per 100000 person years.

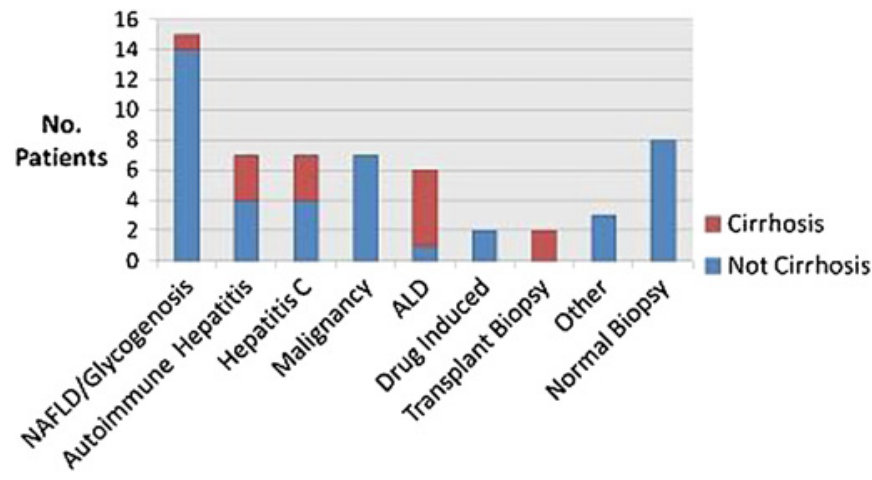

Abstract PTU-026 Figure 1 Index liver biopsy diagnoses.

Conclusion Type 1 Diabetes is associated with significant liver histology abnormalities and a higher than expected occurrence of cirrhosis, portal hypertension and mortality. These findings have implications for long-term management of patients with Type 1 Diabetes.

Competing interests None declared.

\section{REFERENCES}

1. Fleming $\mathbf{K M}$, Aithal GP, Solaymani-Dodaran $\mathbf{M}$, et al. Incidence and prevalence of cirrhosis in the United Kingdom, 1992-2001: a general population-based study. J Hepatol 2008;49:732-8.

2. NHS IC. National Diabetes Audit Mortality Analysis 2007-2008. NHS IC, 2011

\section{PTU-027 LIFETIME ALCOHOL CONSUMPTION IN HEAVY DRINKERS WITH AND WITHOUT LIVER DISEASE. 1: THRESHOLD EFFECT AND MALE-FEMALE DIFFERENCES}

doi:10.1136/gutjnl-2012-302514c.27

${ }^{1} \mathrm{D}$ Gleeson, ${ }^{*} \mathrm{~A}$ Ali, ${ }^{1} \mathrm{~J}$ Jones, ${ }^{1} \mathrm{M}$ Bradley, ${ }^{2} \mathrm{R}$ Peck, ${ }^{3} \mathrm{~K}$ McCormack. ${ }^{1}$ Liver Unit, Sheffield Teaching Hospitals, Sheffield, UK; ${ }^{2}$ Radiology, Sheffield Teaching Hospitals, Sheffield, UK; ${ }^{3}$ Research, Sheffield Teaching Hospitals, Sheffield, UK

Introduction It is unclear whether the risk of alcoholic liver disease (ALD) is related to amount of alcohol consumed (dose effect) or is independent of alcohol consumption above a given threshold (J Hepatol 2004;41:25). The perception that women are more susceptible to ALD than men is based partly on observations that alcohol consumption in women with ALD is less than that of men with ALD. We have previously (Am J Gastro 2008;103:3039) characterised two large cohorts of heavy drinkers ( $>60$ Units/wk (M) or $>40$ Units/wk (F) for $>5$ years): patients with decompensated ALD and controls without serious liver disease on clinical, laboratory and ultrasound examination. Here, we aimed to compare total lifetime alcohol consumption (TLA) between these cohorts and to examine male-female differences in consumption.

Methods Subjects (328 patients, 233 male, mean age $48 \mathrm{yr}$ and 237 heavy-drinking controls, 187 male, mean age $48 \mathrm{yr}$ ) completed a lifetime alcohol questionnaire. Total alcohol consumption was calculated and the predominant beverage group recorded, separately, at home and outside home, and during Monday-Thursday and Friday-Sunday. Data were summed over each stable drinking period in the subject's lifetime. For individual beverage analysis, we assumed that all alcohol consumed in a given circumstance was the 
stated predominant beverage. Body weight data were available in 216 controls and (pre-fluid overload) in 198 patients.

Results TLA was (median (IOR)) $118(80-175) \times 10^{3}$ Units in patients and $131(93-183) \times 10^{3}$ units in controls $(p=0.041)$ by Mann-Whitney): 9 (6-12)-fold and 9 (7-12)-fold higher respectively than the minimum specified by the above inclusion criteria. TLA, corrected for body wt was similar in patients and controls. Females with ALD consumed less total alcohol (absolute 85 (52-11) vs $136(94-193) \times 10^{3}$ units $(p<0.01)$ and corrected for wt $(p=0.04))$, and also, less beer $(p=0.001)$ but more wine $(p<0.001)$ and a trend towards more spirits $(p=0.166)$ over their lifetime than did males with ALD. However, female controls showed similar significant differences from male controls: lower TLA (both absolute $82(69-119)$ vs $145(108-193) \times 10^{3}$ Units) and corrected for body weight) and also, less beer, more wine and more spirits (all comparisons $\mathrm{p} \leq 0.001$ ).

Conclusion The higher total alcohol consumption in controls than in patients is more consistent with a threshold effect then a dose effect of alcohol for development of ALD. Male-female differences in consumption are independent of the presence of liver disease and therefore, are not evidence per se of increased female susceptibility to ALD, although the data do not exclude this.

Competing interests None declared.

\section{PTU-028 TRANSIENT ELASTOGRAPHY IN THE RISK STRATIFICATION OF PATIENTS WITH ABNORMAL LIVER FUNCTION TESTS}

doi:10.1136/gutjnl-2012-302514c.28

E J Britton, * R Saravanan, T Woodhouse, K Koss. Department of Gastroenterology, Macclesfield District General Hospital, Macclesfield, UK

Introduction Transient elastography (TE) (Fibroscan) is a well validated method for assessing liver fibrosis in a trial setting. Gastroenterology Department at Macclesfield DGH is one of the first centres in the UK to adopt Fibroscan examination into the diagnostic pathway of patients with abnormal LFT. This is the first publication of our data of unselected patients undergoing liver stiffness measurement (LSM) demonstrating practicalities and limitations of Fibroscan.

Methods Data were collected from 366 patients with abnormal LFT attending Gastroenterology Clinic from July 2010 to December 2011. Fibroscan examination was performed by two gastroentrology consultants and one specialist nurse. The cohorts of Fatty liver disease (FLD) and Alcoholic liver disease (ALD) were further analysed comparing fibrosis score from TE with clinical findings, laboratory blood tests, ultrasound imaging and liver biopsy.

Results Patients diagnosis were as follows: FLD 35.8\% ( $n=131)$, ALD $14.8 \%(n=54)$, Chronic hepatitis C $15.6 \%(n=57)$, PSC/PBC $7.7 \%(n=28)$, chronic hepatitis B $4.6 \%(n=17)$, haemochromatosis $2.2 \%(n=8)$, AIH $1.4 \%(n=5)$, chronic usage of Methotrexate $1.9 \%$ $(n=7)$. Additionally 42 healthy volunteers participated. The mean time duration for each successful scan was 173 (30-1363) seconds with $18 \%$ of patients requiring the $\mathrm{XL}$ probe due to central obesity. The fibrosis scores recorded for all patients scanned were F0-1 41\% $(n=150)$, F2 6.3\% $(n=23)$, F3 7.1\% $(n=26)$ and F4 26\% $(n=95) .15 \%$ of scans were considered to be failed as LSM/IOR was $>33$, most prevalent in the FLD group $16.8 \%(n=22)$ and wasn't operator or probe dependent. Significant proportion of patients with liver fibrosis (F3-4) had no clinical signs of chronic liver disease. $80 \%$ of patients F3-F4 fibrosis demonstrated portal hypertension on imaging and $32 \%$ of patients in that group had low platelets
(3. The sensitivity and specificity of low platelets for significant fibrosis was $32 \%$ and $91 \%$ and for portal hypertension on imaging was $64 \%$ and $89 \%$ respectively. There was significant correlation between liver fibrosis scoring on TE and Metvir fibrosis scoring on biopsy in patients without high degree of inflammation. However, coexisting acute inflammation in some cases contributed to false positive results on Fibroscan.

Conclusion The Fibroscan technology has allowed rapid stratification of patients with chronic liver diseases. Significant proportion of patients (40\%) were appropriately reassured without need of undergoing liver biopsy. $20 \%$ of patients without stigmata of chronic disease and without conventional laboratory markers of liver fibrosis were diagnosed with liver cirrhosis. Early experience has shown difficulties of performing TE in patients with central obesity and FLD. Fibroscan finds its valuable role in the care pathway of patients with abnormal LFT.

Competing interests None declared.

\section{PTU-029 A NOVEL MRI PROTOCOL TO EXAMINE HAEMODYNAMIC COMPARTMENTS IN COMPENSATED LIVER CIRRHOSIS}

doi:10.1136/gutjnl-2012-302514c.29

${ }^{1} \mathrm{E} F$ Cox, ${ }^{*}{ }^{2} \mathrm{~A}$ Ghezzi, ${ }^{2} \mathrm{M}$ Patel, ${ }^{2} \mathrm{~A}$ Jackson, ${ }^{1} \mathrm{C}$ Costigan, ${ }^{2} \mathrm{M}$ W James, ${ }^{2} \mathrm{~S} D$ Ryder, ${ }^{1} \mathrm{P}$ A Gowland, ${ }^{2} \mathrm{G}$ P Aithal, ${ }^{1} \mathrm{~S}$ T Francis, ${ }^{2} \mathrm{~N}$ Guha. ${ }^{1}$ Sir Peter Mansfield Magnetic Resonance Centre, University of Nottingham, Nottingham, UK; ${ }^{2}$ NIHR Nottingham Digestive Diseases Biomedical Research Unit, Nottingham University Hospitals, Nottingham, UK

Introduction The hyperdynamic circulation in cirrhosis results from changes in the splanchnic, systemic, cardiac and renal compartments; it underpins the clinical consequences of portal hypertension. We present a novel MRI protocol that provides non-invasive measurement of these different haemodynamic compartments in a single assessment. Phase contrast (PC) MRI is a validated technique for the measurement of velocity (in $\mathrm{cm} / \mathrm{s}$ ), area, and hence flux (=velocity*area (in $\mathrm{ml} / \mathrm{s})$ ) in a given vessel. ${ }^{1}$ Here, we use PC-MRI, together with MR measures of cardiac function, to assess haemodynamics in early, compensated cirrhosis and matched healthy volunteers.

Methods Patients were studied from a prospective, longitudinal study of compensated cirrhosis (CC) (Child Pugh A) with age and sex matched healthy volunteers (HV). 20 CC patients (13M/7F, age $57 \pm 1$ y (mean \pm SEM), aetiology 2 ALD, 1 HBV, 7 HCV, 3 NAFLD, 1 PBC, 1 Haemochromatosis, MELD 0-10.6) and $20 \mathrm{HV}$ : 13M/7F, age $57 \pm 2$ y were included. PC-MRI data were collected for the superior mesenteric (SMA), splenic (SA), hepatic (HA), and renal (RA) artery and portal vein (PV). 15 phases were acquired across the cardiac cycle to calculate the mean area, velocity and flux for each vessel. Cardiac MR consisted of short axis cine images to measure left ventricular ejection fraction (EF) and PC-MRI of the aorta. All data were collected within $20 \mathrm{~min}$, with images acquired during short breath-holding (12-15 s).

Results In CC, the portal vein was dilated and had a reduction in velocity compared to the HV group (Abstract PTU-029 figure 1A). The SMA (Abstract PTU-029 figure 1B) and SA, showed vasodilation and increased flow velocity, and a resulting increase in flux in patients with CC compared to the HV. There was a trend for a reduction in RA velocity (Abstract PTU-029 figure 1C) and an increase in HA velocity in CC. The ejection fraction was significantly higher in CC compared with the HV (Abstract PTU-029 figure 1D). There was no change in aortic flux, velocity or area with CC. 